\title{
Erratum to: Non-singular rotating black hole with a time delay in the center
}

\author{
Tommaso De Lorenzo ${ }^{1}$ • Andrea Giusti ${ }^{1,2}$. \\ Simone Speziale ${ }^{1}$
}

\section{Erratum to: Gen Relativ Gravit (2016) 48: 31 DOI 10.1007/s10714-016-2026-5}

The original version of this article unfortunately contained a mistake. The complete last name of the first author is De Lorenzo, the first name is Tommaso.

The online version of the original article can be found under doi:10.1007/s10714-016-2026-5.

$\triangle$ Simone Speziale simone.speziale@cpt.univ-mrs.fr

1 Centre de Physique Theorique, CNRS-UMR 7332, Aix-Marseille Université \& Université de Toulon, Case 907, Campus de Luminy, 13288 Marseille, France

2 Dip. di Fisica e Astronomia, Università di Bologna, via Irnerio 46, 40126 Bologna, Italy 